\title{
Upregulation of NOX2 and NOX4 Mediated by TGF- $\beta$ Signaling Pathway Exacerbates Cerebral Ischemia/Reperfusion Oxidative Stress Injury
}

\author{
Zheng Lou ${ }^{\mathrm{a}}$ Ai-Ping Wang ${ }^{\mathrm{e}}$ Xiao-Ming Duan ${ }^{\mathrm{b}}$ Guo-Huang Hub $\mathrm{u}^{\mathrm{b}, \mathrm{c}}$ \\ Gui-Lin Song ${ }^{\mathrm{b}, \mathrm{d}}$ Mei-Ling Zuo ${ }^{\mathrm{b}}$ Zhong-Bao Yang ${ }^{\mathrm{b}, \mathrm{d}}$
}

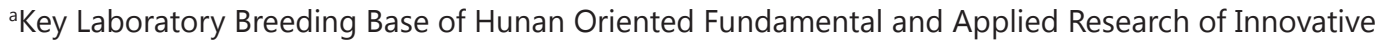
Pharmaceutics, Changsha Medical University, Changsha, 'b The Affiliated Changsha Hospital of Hunan Normal University, Changsha, 'Institute of Digestive Surgery of Changsha, Institute of Emergency and Critical Care Medicine of Changsha, eInstitue of Clinical Medicine, Nanhua Affiliated Hospital, University of South China, Hengyang, China

\section{Key Words}

Cerebral ischemia/reperfusion injury • TGF- $\beta$ signaling • ALK5 • NOX2 • NOX4 • ROS

\begin{abstract}
Background/Aims: Ischemic stroke is still one of the leading debilitating diseases with high morbidity and mortality. NADPH oxidase (NOX)-derived reactive oxygen species (ROS) play an important role in cerebral ischemia/reperfusion (I/R) injury. However, the mechanism underlying the regulation of ROS generation is still not fully elucidated. This study aims to explore the role of transforming growth beta (TGF- $\beta$ ) signals in ROS generation. Methods: Sprague-Dawley rats were subjected to I/R injury, and PC-12 cells were challenged by hypoxia/reoxygenation (H/R) and/or treated with activin receptor-like kinase (ALK5) inhibitor Sb505124 or siRNA against ALK5. Brain damage was evaluated using neurological scoring, triphenyl tetrazolium chloride staining, hematoxylin and eosin staining, infarct volume measurement, TUNEL staining, and caspase- 3 activity measurement. Expression of TGF- $\beta$ and oxidative stress-related genes was analyzed by real-time polymerase chain reaction and Western blot; NOX activity and ROS level were measured using spectrophotometry and fluorescence microscopy, respectively. Results: I/R contributed to severe brain damage (impaired neurological function, brain infarction, tissue edema, apoptosis), TGF- $\beta$ signaling activation (upregulation of ALK5, phosphorylation of SMAD2/3) and oxidative stress (upregulation of NOX2/4, rapid release of ROS [oxidative burst]). However, Sb505124 significantly reversed these alterations and protected rats against I/R injury. As in the animal results, $H / R$ also contributed to TGF- $\beta$ signaling activation and oxidative stress. Likewise, the inhibition of ALK5 or ALK5 knockdown significantly reversed these alterations in PC-12 cells. Other than ALK5 knockdown, ALK5 inhibition had no effect
\end{abstract}

Z. Lou and A.-P. Wang contributed equally to this work. 
on the expression of ALK5 in PC-12 cells. Conclusions: Our studies demonstrated that TGF- $\beta$ signaling activation is involved in the regulation of NOX2/NOX4 expression and exacerbates cerebral I/R injury.

\section{Introduction}

Ischemic stroke is still one of the most devastating neurological diseases with high morbidity and mortality $[1,2]$. Blood flow obstruction or insufficient blood supply is the fundamental cause of ischemic stroke, which contribute to reduced cerebral supply of oxygen and glucose, and subsequently triggers a cascade of pathological events such as excitotoxicity, inflammatory response, and oxidative stress, ultimately leading to irreversible neuronal injury [3]. Among these pathophysiological events, oxidative stress is the most common injury that occurs during the process of ischemia/reperfusion (I/R) injury, and has become a major challenge in brain injury treatment $[4,5]$. Previous studies have found that the main cause of oxidative stress is accumulation of reactive oxygen species (ROS). Although numerous biological processes are involved in ROS generation, NADPH oxidase (NOX) was demonstrated to be the main enzyme responsible for ROS generation. In mammals, seven NOX family members-Nox1 to Nox5, dual oxidase (Duox)-1, and Duox-2have been demonstrated in tissues [6, 7]. However, our previous studies found that NOX2 and NOX4 were the major subtypes responsible for brain tissue ROS production in a rat model of ischemic stroke [8]. Presently, many factors have been reported to be involved in the regulation of NOX expression, including transcription factor NF- $\mathrm{KB}$ and myosin light chain [9]. However, the detailed mechanism underlying NOX expression regulation is not fully elucidated.

Transforming growth factor $\beta$ (TGF- $\beta$ ) is a member of a large family of pleiotropic cytokines that are involved in many biological processes, including growth control, differentiation, migration, cell survival, adhesion, and apoptosis [10]. Previous studies have reported that TGF- $\beta$ signaling is involved in tumor suppression [11] and ROS production [12]. Through binding to its receptors (serine/threonine kinases), TGF- $\beta$ triggers a cascade of events such as ERK signaling pathway activation and mTOR mediated protein synthesis [11]. Furthermore, these processes are usually involved in SMAD2/3 phosphorylation, which is a possible consequence of the action of activin receptor-like kinase 5 (ALK5) [13]. Emerging evidence has demonstrated that TGF- $\beta$ signaling is crucial in the pathogenesis of several central nervous system (CNS) disorders, such as neurodegenerative disorders [14, 15]. Especially, in brain tissue, increased TGF- $\beta$ levels were correlated with cerebral ischemic injury [16]. However, very few studies have been conducted to explore the relationship between TGF- $\beta$ signaling and oxidative stress in ischemic stroke.

To investigate the mechanism of oxidative stress after ischemic stroke, Sprague-Dawley (SD) rats were subjected to I/R injury and PC-12 cells were subjected to H/R injury to mimic ischemic stroke; an ALK5 inhibitor or siRNA against ALK5 were used to observe the role of ALK5 in oxidative stress. We found that oxidative stress after I/R injury was associated with TGF- $\beta$ signaling activation. To our knowledge, this is the first study to explore the role of TGF- $\beta$ signaling in NOX-mediated oxidative stress in cerebral I/R injury, and aims to provide novel targets for ischemic stroke therapy.

\section{Materials and Methods}

\section{Animal experiment}

Male SD rats weighing 250-300g purchased from Hunan SJA Laboratory Animal Co., Ltd. (Hunan, China). Animals were housed for a week to acclimate to the environment. Before experiments, rats were fasted for $24 \mathrm{~h}$ with free access to drinking water. The study was performed following the Guide for the Care and Use of Laboratory Animals, published by the National Institutes of Health (NIH Publication No. 
85-23, revised 1996) and experiments were approved by the Hunan Normal University Veterinary Medicine Animal Care and Use Committee.

The rat model of I/R injury was established according to the method previously reported from in our laboratory ${ }^{[15]}$. Briefly, a 4/0 surgical nylon monofilament with rounded tip was introduced into the left internal carotid artery through the external carotid stump, and advanced 20-21 mm past the carotid bifurcation until a slight resistance was felt. At this point, the intraluminal filament blocked the origin of the middle cerebral artery and occluded all sources of blood flow from the internal carotid artery, anterior cerebral artery, and posterior cerebral artery. The filament was left in place for $2 \mathrm{~h}$ and then withdrawn for reperfusion for $24 \mathrm{~h}$. The body temperature of the rat was maintained at approximately $37{ }^{\circ} \mathrm{C}$ throughout the procedure. Animals from the sham group underwent the same procedure except that the occluding filament was inserted only $7 \mathrm{~mm}$ above the carotid bifurcation.

The animals were randomly allocated to four groups ( $n=8$ per group): the sham group (underwent surgical procedures without ischemic insult), the I/R group (subjected to $2 \mathrm{~h}$ of ischemia followed by $24 \mathrm{~h}$ of reperfusion), the Sb505124 intervention group (subjected to I/R with Sb505124 (15 mg/kg)), and the vehicle group (subjected to I/R with saline). At the end of reperfusion, neurological deficit score was assessed first, and then the brain tissue was harvested for infarct volume measurement or other measurements (expression of mRNA or protein).

\section{Cell culture}

The PC-12 cell line was purchased from the Committee on Type Culture Collection of Chinese Academy of Sciences of ShangHai. Cells were cultured in DMEM medium supplemented with 10\% FBS and penicillin/ streptomycin in 95\% air and 5\% $\mathrm{CO}_{2}$. Cells were subcultured and seeded into 6- and 24-well plates for miRNA mimic and inhibitor functional assays, respectively. Finally, cells were digested with $0.2 \%$ trypsinogen and collected for mRNA or protein analysis.

\section{Cell model of $\mathrm{H} / \mathrm{R}$}

To establish the H/R model, PC-12 cells were subjected to $5 \mathrm{~h}$ of hypoxia $\left(\mathrm{N}_{2} / \mathrm{CO}_{2}, 95: 5\right)$ in preconditioned hypoxic medium (serum-free DMEM without glucose and sodium pyruvate, with incubation under hypoxic conditions for $2 \mathrm{~h}$ ) followed by $20 \mathrm{~h}$ of reoxygenation. Hypoxic medium was replaced with fresh medium upon switching to reoxygenation.

\section{Assessment of neurological deficit score and measurement of infarct volume}

Neurological scoring was performed to evaluate impaired neurobiological function by using the following 5-point rating scale: $0=$ no deficit, $1=$ failure to extend the left forepaw, $2=$ decreased grip strength of left forepaw, 3 = circling to the left on pulling the tail, 4 = spontaneous circling.

For infarct volume measurement, brains were sectioned into 4 coronal sections of $0.2-0.3 \mathrm{~cm}$ thickness. Sections were immersed in $2 \%$ 2, 3,5-tripenyltetrazolium chloride (TTC) for 30 min at $37{ }^{\circ} \mathrm{C}$ and then scanned into a computer and evaluated with imaging analysis software (Image J, National Institutes of Health, Bethesda, MD). The presence or absence of infarction was determined by examining TTC staining. Infarct volume $\left(\mathrm{cm}^{3}\right)$ for each section was equal to infarct area $\left(\mathrm{cm}^{2}\right)$ multiplied by section thickness $(0.2$ or $0.3 \mathrm{~cm}$ ). Total infarct volume for each brain was then calculated by summing the infarct volumes of all sections. To minimize the effect of edema on the accuracy of infarct volume measurement, the final infarct volume was corrected by applying the following equation: corrected infarct volume = total infarct volume left hemisphere volume/right hemisphere volume. Here, left hemisphere refers to the non-ischemic hemisphere of the brain while right hemisphere refers to ischemic contralateral side.

\section{Determination of TGF- $\beta 1$ level}

Measurement of TGF- $\beta 1$ level in brain tissues was performed with a commercial ELISA kit. Briefly, the whole process was conducted as follows: preparing standards and samples, adding prepared standards $\left(50 \mu \mathrm{L} /\right.$ well) and incubating for $30 \mathrm{~min}\left(37^{\circ} \mathrm{C}\right)$, washing and adding enzyme-labeled reagent $(50 \mu \mathrm{L} /$ well $)$ and incubating for $30 \mathrm{~min}\left(37^{\circ} \mathrm{C}\right)$, washing and developing, ending development, reading at $450 \mathrm{~nm}$, and calculating the final values.

\section{KARGER}


Lou et al.: A New Mechanism for NOX2 and NOX4 Expression Regulation in the Process of Cerebral Ischemia/Reperfusion Injury

ALK5 inhibition or knockdown

To investigate the role of TGF- $\beta$ signaling in ROS generation, PC-12 cells were treated with ALK5 inhibitor. Briefly, PC-12 cells were seeded into 6-well plates with culture medium containing 10\% FBS until $70 \%$ confluence, and the cells were then incubated with serum-free culture medium supplemented with 50 nM Sb505124 for $6 \mathrm{~h}$.

To further confirm that ALK5 mediates NOX expression regulation, small interfering RNA (siRNA) against ALK5 was designed and synthesized. Before the intervention experiment, a mix of Lipofectamine ${ }^{\circledR}$ 2000 and ALK5 siRNA or negative control siRNA were prepared. Then, the mix was added into cell plates for $6 \mathrm{~h}$ of transfection according to the manufacturer's instructions.

Finally, cells were collected for mRNA, protein, and other analyses.

\section{Real-time PCR}

Real-time PCR was used to analyze ALK5, NOX2, and NOX4 mRNA levels in brain tissue. Total RNA was extracted by using TRIzol reagent (TakaRa Biotechnology Co., Ltd., Dalian, China) and the concentration and purity of RNA was determined spectrophotometrically. Briefly, $200 \mathrm{ng}$ of RNA from each sample was used for reverse transcription by using a transcription Kit (DRR037A; TaKaRa). Real-time PCR was used to quantitatively determine ALK5, NOX2, and NOX4 mRNA expression levels with SYBR Premix Ex Taq (TaKaRa) using the ABI 7300 Real-Time PCR System. PCR primers for ALK5, NOX2, NOX4, and $\beta$-actin are shown in Table 1. Data analysis was performed with the comparative Ct method by using the ABI software. Results were adjusted with the ratio of TGF- $\beta$, NOX2, and NOX4 mRNA to $\beta$-actin mRNA.

Western blot

Total protein for each sample was extracted using a commercial kit. Briefly, $40 \mu \mathrm{g}$ protein was used for Western blot analysis as follows: SDS-PAGE (on 10\% gel), transfer to polyvinylidene fluoride membrane, incubation with rabbit anti-pSMAD2/3, -NOX2, and -NOX4 primary antibodies (Santa Cruz Biotechnology, Santa Cruz, CA) followed by horseradish peroxidase-conjugated secondary antibodies, and determination of band signals using enhanced chemiluminescence (BeyoECL Plus Kit, Amersham Biosciences, Piscataway, NJ) and the Molecular Imager ChemiDoc XRS System (Bio-Rad, Philadelphia, PA). Densitometric analysis was performed with Image J 1.43 (National Institutes of Health). Loading control was normalized to $\beta$-actin (Millipore, Billerica, MA).

Measurement of activities of NOX and caspase-3

NOX activity was measured using a commercially available kit (Genmed Pharmaceutical Technology Co., Ltd., Shanghai, China) following the manufacturer's instructions. Briefly, supernatant of cell lysates was incubated with oxidized cytochrome $\mathrm{C}$ in a quartz cuvette at $30^{\circ} \mathrm{C}$ for $3 \mathrm{~min}$, and then the NOX substrate (NADPH) was added to the reaction mixture and incubated for $15 \mathrm{~min}$. The change in absorbance at $550 \mathrm{~nm}$ was monitored by a spectrophotometer. NOX activity was estimated by calculating cytochrome c reduction per min.

The procedure for measurement of caspase-3 activity was carried out with a kit following the manufacturer's instructions (Beyotime, Shanghai, China). In brief, $10 \mu \mathrm{L}$ of cell lysate was mixed with $90 \mu \mathrm{L}$ of working solution containing caspase-3 substrate (Ac-DEVD-pNA) and then the mixture was incubated at $37^{\circ} \mathrm{C}$ for $60 \mathrm{~min}$. Absorbance was recorded at $405 \mathrm{~nm}$. Enzyme activity was presented as U/g protein and $1 \mathrm{U}$ was defined as the amount of enzyme required to cleave $1.0 \mathrm{nmol}$ of Ac-DEVD-pNA per hour at $37^{\circ} \mathrm{C}$.

Determination of ROS levels

Measurement of intracellular ROS levels was dependent on the fluorescent signal of 2',7'-dichlorodihydrofluorescein diacetate (DCFH-DA), a cell-permeable indicator of ROS (Beyotime,

Table 1. Primers for real-time PCR

\begin{tabular}{lrrc}
\hline Gene & Forward primer & Reverse primer & Product size (bp) \\
\hline NOX2 & 5'-ACAAGGTTTATGACGATGAGCC-3' & 5'-TTGAGCAACACGCACTGGAA-3' & 174 \\
NOX4 & 5'-CTGACAGGTGTCTGCATGGT-3' & 5'-ACTTCAACAAGCCACCCGAA-3' & 160 \\
ALK5 & 5'-TTGTTGAGGAGAAGCTGAGGC-3' & 5'-CACTGTAATGCCTTCGCCCC-3' & 154 \\
$\beta$-actin & 5'-CCCATCTATGAGGGTTACGC-3' & 5'-TTTAATGTCACGCACGATTTC-3' & 150 \\
\hline
\end{tabular}


Lou et al.: A New Mechanism for NOX2 and NOX4 Expression Regulation in the Process of Cerebral Ischemia/Reperfusion Injury

Shnanghai, China). DCFH-DA is nonfluorescent until the acetate groups are removed by intracellular ROS. Briefly, PC-12 cells were washed with PBS and incubated with DCFH-DA (10 $\mu \mathrm{M})$ at 37 ? for 20 min. Then, ROS-mediated fluorescence was observed under a fluorescent microscope with excitation set at $502 \mathrm{~nm}$ and emission set at $523 \mathrm{~nm}$. Results expressed as arbitrary units.

\section{Statistical analysis}

SPSS software (Version 11; SPSS, Inc., Chicago, IL) was used for statistical analysis. Data were expressed as mean \pm SEM. Differences in measured values among the multiple groups were analyzed by analysis of variance with Bonferroni's multiple comparison test. One-way analysis of variance was used to compare the data distribution between groups. Differences were considered significant when $\mathrm{P}<0.05$.

\section{Results}

\section{Inhibition of ALK5 alleviated cerebral ischemia/reperfusion injury}

TGF- $\beta$ signaling has many biological functions in animals. To explore whether TGF- $\beta$ signaling plays an important role in cerebral I/R injury, we developed an SD rat model of ischemic stroke and intervened with ALK5 inhibitor Sb505124. We found that rats subjected to I/R injury exhibited significantly impaired neurological function (Fig. 1A), brain infarction (TTC staining white and infarct volume up to $0.4 \mathrm{~cm}^{3}$ ) (Fig. 1B and C), morphological alterations in brain tissue (shrinking and edema) (Fig. 1D), and apoptosis (positive TUNEL staining and increased caspase-3 activity) (Fig. 1E and F). These findings indicated that these rats subjected to I/R injury had sustained obvious brain injury and inactivation of some metabolic enzymes that are essential for proper brain function. However, the ALK5 inhibitor Sb505124 significantly alleviated cerebral ischemia/reperfusion injury, improved neurological function (Fig. 1A), decreased brain tissue infarct volume (Fig. 1B and C), inhibited brain tissue morphological alteration (Fig. 1D), and decreased apoptosis (Fig. 1E and F). These data suggested that ALK5 is involved in and plays an important role in I/R injury.

Inhibition of ALK5 decreased expression of NOX2/4 and ROS generation after cerebral ischemia/reperfusion

Oxidative stress is an important pathophysiological process during I/R injury. To further observe whether TGF- $\beta$ signaling has any effect on cerebral I/R oxidative stress injury, the expression of NOX2, NOX4 (the main oxidative stress-related enzymes in brain tissue),

Fig. 1. Inhibition of ALK5 alleviated cerebral ischemia/reperfusion injury. A: Neurological deficit score in each group ( $\mathrm{n}=8)$; B: Representative image of triphenyltetrazolium chloride-stained brain tissue from each group (red area represents non-infarction, white area represents the infarction); C: Infarct volume in each group ( $\mathrm{n}=8$ ); D: Representative image of HEstained brain tissue from each group; E: Representative image of TUNEL-stained brain tissue from each group; F: caspase 3 activity. All values are expressed as the mean \pm S.E.M. I/R: ischemia/reperfusion; +Sb505124: I/R+ Sb505124 (15 mg/kg); +vehicle: $\mathrm{I} / \mathrm{R}+$ saline; ${ }^{*} \mathrm{P}<0.001$ vs sham $;{ }^{* * *} \mathrm{P}<0.001$ vs sham; ${ }^{\#} \mathrm{P}<0.05$ vs I/R.

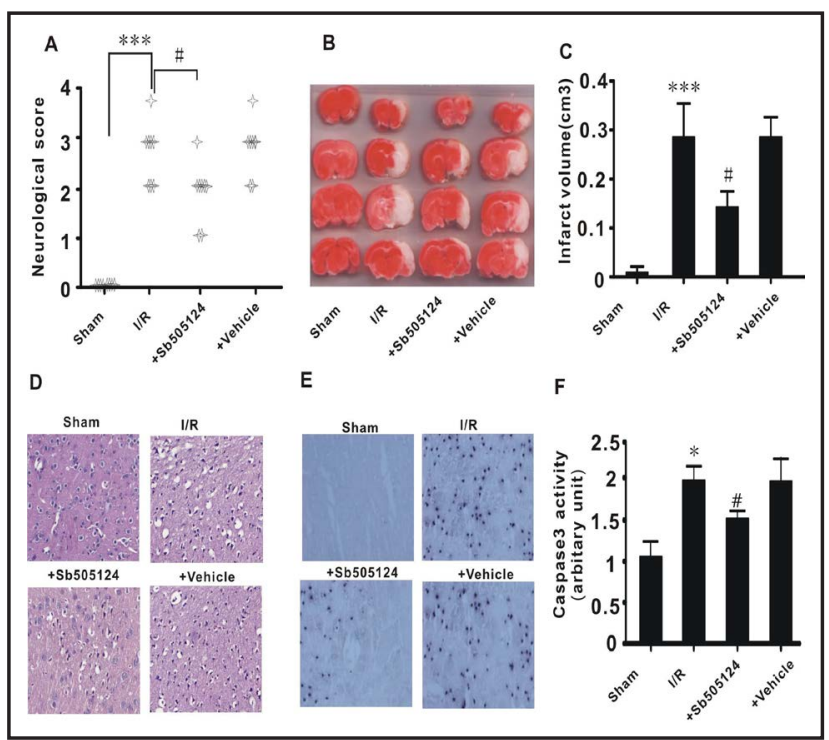


total NOX activity, as well as ROS level were measured. We found that cerebral $\mathrm{I} / \mathrm{R}$ injury contributed to increased expression of NOX2 and NOX4 (mRNA and protein) (Fig. 2A-D), higher total NOX enzyme activity (Fig. 2E), and increased ROS level (Fig. 2F) compared with brain tissue in the sham group. However, the ALK5 inhibitor Sb505124 significantly inhibited NOX2 and NOX4 expression (mRNA and protein), decreased total NOX enzyme activity, and reduced ROS level compared with the I/R group (Fig. 2A-F). These results suggested an important role for ALK5 in cerebral I/R oxidative stress injury.

Effect of ALK5 inhibition on TGF- $\beta 1$ level and TGF- $\beta$ signaling after cerebral ischemia/reperfusion

To investigate the relationship between TGF- $\beta$ signaling and cerebral I/R injury, TGF- $\beta 1$ level was determined; ALK5 expression (mRNA and protein), and the phosphorylation of SMAD2/3 were measured. We found that brain tissue with I/R injury had higher TGF- $\beta 1$ levels (Fig. 3A). We also found that ALK5 expression (mRNA and protein) and SMAD2/3 phosphorylation significantly increased in tissues subjected to I/R injury compared with the sham group (Fig. 3BF). These findings indicate that cerebral I/R injury was associated with activation of TGF- $\beta$ signaling. Expectedly, ALK5 inhibition with Sb505124 significantly decreased TGF- $\beta 1$ levels, inhibited ALK5 expression (mRNA and protein), and decreased SMAD2/3 phosphorylation (Fig. 3A-F). This suggested a key role for TGF- $\beta$ signaling in cerebral I/R injury and a vital role for ALK5 in TGF- $\beta$ signaling.

Effect of ALK5 inhibition on TGF- $\beta$ signaling, NOX2/4 expression and ROS generation in $P C-12$ cells after $H / R$

To further investigate the role of TGF- $\beta$ signaling in oxidative stress injury, PC-12 cells were subjected to H/R injury followed by intervention using ALK5 inhibition with Sb505124. We found that ALK5 (Fig. 4A-B), NOX2, (Fig. 4A, F-G), and NOX4 (Fig. 4H-I) expression (mRNA and protein), SMAD2/3 phosphorylation (Fig. 4C-E), total NOX enzyme activity (Fig. 4J), and ROS level (Fig. 4K) significantly increased

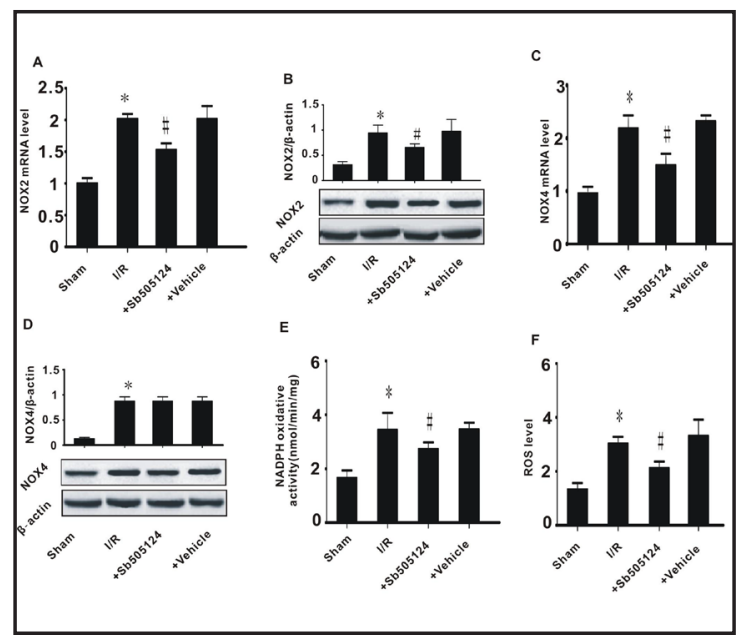

Fig. 2. Inhibition of ALK5 decreased expression of NOX2/4 and ROS generation after cerebral ischemia/ reperfusion. A: NOX2 mRNA level; B: NOX2 protein level; C: NOX4 mRNA level; D: NOX4 protein level; E: NOX activity; F: ROS level. All values are expressed as the mean \pm S.E.M. I/R: ischemia/reperfusion; +Sb505124: I/R + Sb505124 (15 mg/kg); +vehicle: I/R + saline; ${ }^{*} \mathrm{P}<0.001$ vs sham; ${ }^{* *} \mathrm{P}<0.001$ vs sham; ${ }^{*} \mathrm{P}<0.05$ vs I/R.

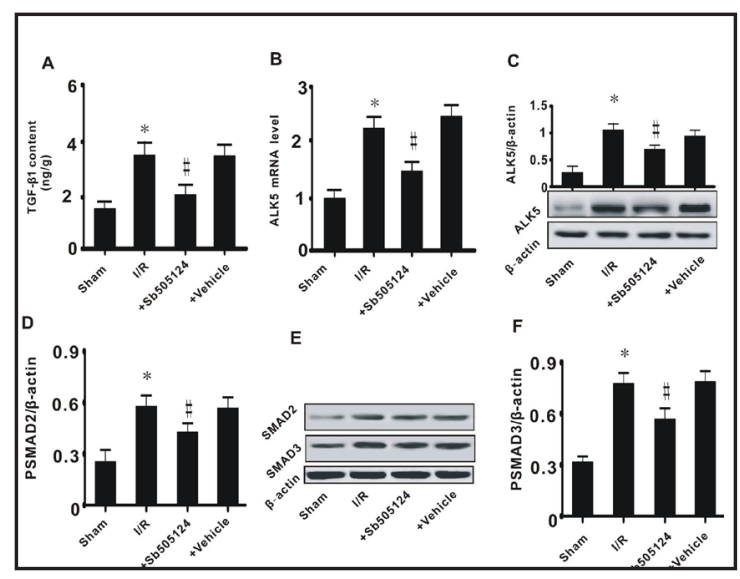

Fig. 3. Effect of ALK5 inhibition on TGF- $\beta 1$ level and TGF- $\beta$ signaling after cerebral ischemia/reperfusion. A: TGF- $\beta 1$ level; B: ALK5 mRNA level; C: ALK5 protein level; D: Ratio of phosphorylated SMAD2 to $\beta$-actin; E: Protein level of phosphorylated SMAD2/3; F: Ratio of phosphorylated SMAD3 to $\beta$-actin. All values are expressed as mean \pm S.E.M. I/R: ischemia/reperfusion; +Sb505124: I/R + Sb505124 (15 mg/kg); +vehicle: I/R + saline; ${ }^{*} \mathrm{P}<0.001$ vs sham; ${ }^{\mathrm{P}} \mathrm{P}<0.05$ vs I/R. 
in cells subjected to $\mathrm{H} / \mathrm{R}$ compared with controls. This demonstrated that there was a close relationship between TGF- $\beta$ signaling and oxidative stress. Notably, as in the animal experiments, the ALK5 inhibitor Sb505124 significantly decreased NOX2 (Fig. 4A, F-G) and NOX4 (Fig. 4H-I) expression (mRNA and protein), SMAD2/3 phosphorylation (Fig. 4CE), total NOX enzyme activity (Fig. 4J), and ROS level (Fig. 4K), respectively. However, the ALK5 inhibitor had no effect on ALK5 expression (mRNA and protein) (Fig. 4A-B). These results indicated that $\mathrm{H} / \mathrm{R}$ contributed to ROS generation and was associated with increased NOX2/4 expression; however, this process was not involved in increased ALK5 expression but in increased SMAD2/3 phosphorylation.

\section{Effect of ALK5 knockdown on TGF- $\beta$ signaling, NOX2/4 expression, and ROS generation in} $P C$ - 12 cells after $H / R$

We previously demonstrated that $H / R$ contributed to activation of TGF- $\beta$ signaling and ultimately to the excessive production of ROS. Our next experiments were designed to further investigate the role of TGF- $\beta$ signaling in oxidative stress. We used siRNA against

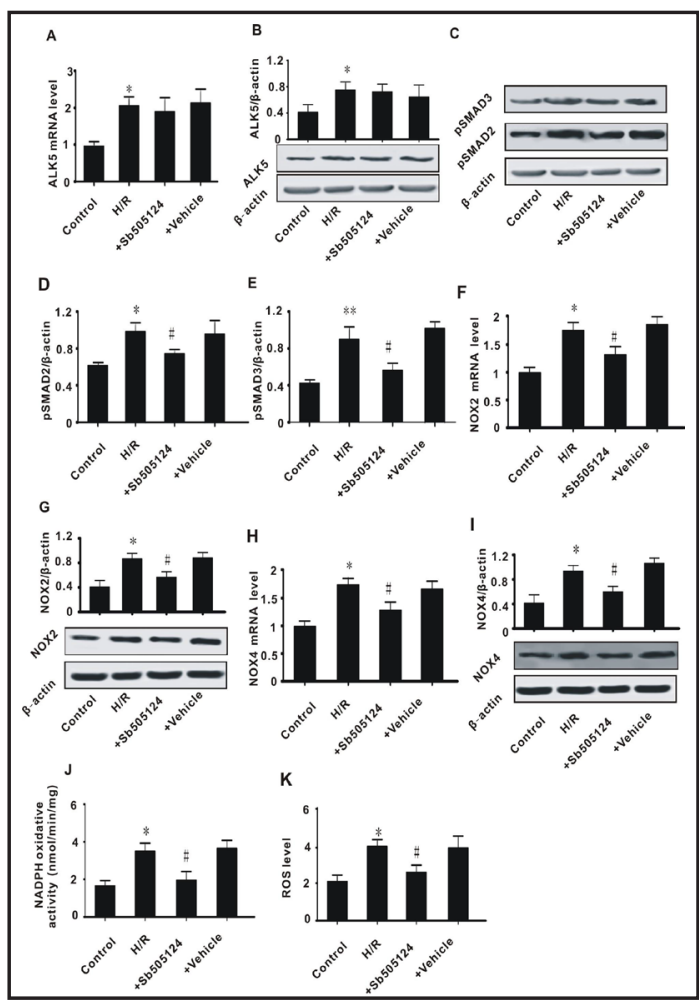

Fig. 4. Effect of ALK5 inhibition on TGF- $\beta$ signaling, NOX2/4 expression, and ROS generation in PC12 cells after H/R. A: ALK5 mRNA level; B: ALK5 protein level; C: Protein level of phosphorylated SMAD2/3;D: Ratio of phosphorylated SMAD2 to $\beta$-actin; E: Ratio of phosphorylated SMAD3 to $\beta$-actin; F: NOX2 mRNA level; G: NOX2 protein level; H: NOX4 mRNA level; I: NOX4 protein level; J: NOX activity; K: ROS level. All values are expressed as the mean \pm S.E.M. H/R: hypoxia/reperfusion; + control siRNA: $\mathrm{H} / \mathrm{R}+$ negative control siRNA; +ALK5 siRNA: H/R + siRNA against ALK5; +vehicle: $\mathrm{H} / \mathrm{R}+$ dimethyl sulfoxide (DMSO). ${ }^{*} \mathrm{P}<0.05$ vs control; ${ }^{* *} \mathrm{P}<0.01$ vs control; ${ }^{\#} \mathrm{P}<0.05$ vs $\mathrm{H} / \mathrm{R}$.

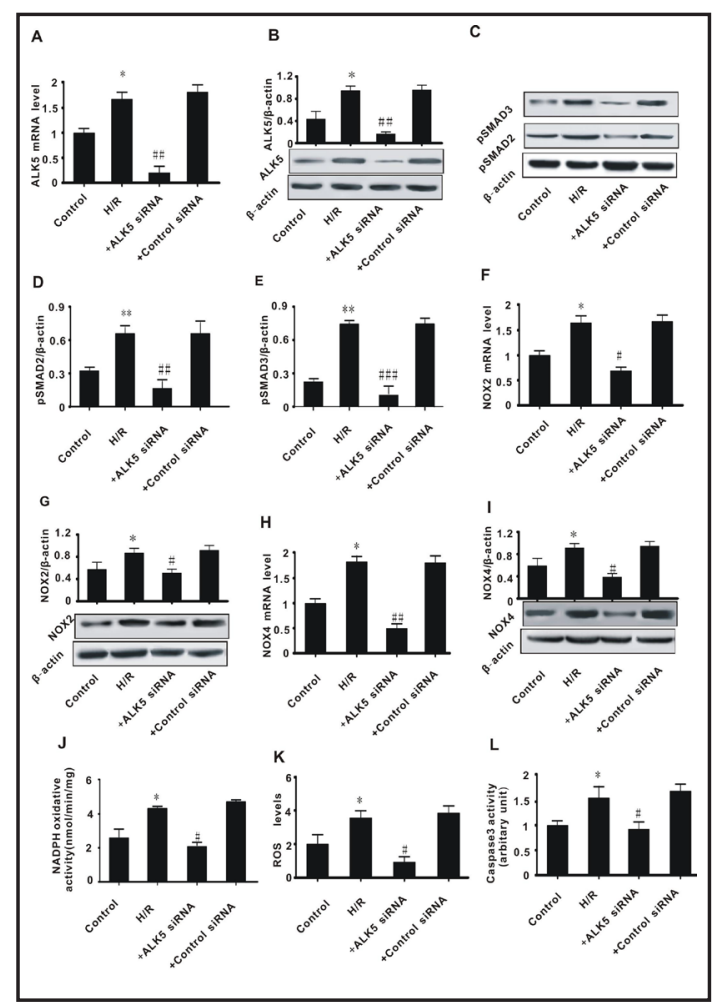

Fig. 5. Effect of ALK5 knockdown on TGF- $\beta$ signaling, NOX2/4 expression, and ROS generation in PC-12 cells after H/R. A:ALK5 mRNA level; B: ALK5 protein level; C: Protein level of phosphorylated SMAD2/3; D: Ratio of phosphorylated SMAD2 to $\beta$-actin; E: Ratio of phosphorylated SMAD3 to $\beta$-actin; F: NOX2 mRNA level; G: NOX2 protein level; H: NOX4 mRNA level; I: NOX4 protein level; J: NOX activity; K: ROS level; L: Caspase-3 activity. All values are expressed as the mean \pm S.E.M. H/R: hypoxia/reperfusion; +control siRNA: H/R + negative control siRNA; +ALK5 siRNA: H/R + siRNA against ALK5.; +vehicle: $\mathrm{H} / \mathrm{R}+\mathrm{DMSO} .{ }^{*} \mathrm{P}<0.05$ vs control; ${ }^{* *} \mathrm{P}<0.01$ vs control; "P $<0.05$ vs $H / R$; ;\# $\mathrm{P}<0.01$ vs $H / R$; ;\#\# $\mathrm{P}<0.001$ vs $\mathrm{H} / \mathrm{R}$..

\section{KARGER}


ALK5 to knockdown ALK5 and observed the effect on PC-12 cells subjected to H/R. We found that siRNA against ALK5 significantly decreased ALK5 expression (mRNA and protein) in cells subjected to $H / R$, but cells treated with negative control siRNA showed no significant effect on ALK5 expression, indicating the effect of ALK5 siRNA knockdown (Fig. 5A and B). We also found that ALK5 knockdown significantly decreased pSMAD2/3 phosphorylation (Fig. 5C-E), NOX2 and NOX4 expression (mRNA and protein) (Fig. 5F-I), total NOX enzyme activity (Fig. 5J), and ROS level (Fig. 5K), as well as caspase-3 activity (Fig. 5L), compared with H/R. This suggested that ALK5 is a key modulator of TGF- $\beta$ signaling, which involves in the regulation of expression of the oxidative stress-related genes NOX 2/4.

\section{Discussion}

Our results demonstrated that TGF- $\beta$ signaling is involved in the regulation of NOX $2 / 4$ expression in brain tissue subjected to I/R injury. The in vivo results showed that $\mathrm{I} / \mathrm{R}$ contributed to increased ALK5 and NOX2/4 expression, SMAD2/3 phosphorylation, and ROS level, concomitantly with increasing severity of brain damage. However, treatment with the ALK5 inhibitor significantly reversed these alterations. This suggested a close relationship between TGF- $\beta$ signaling activation and oxidative stress. Consistent with the animal results, $\mathrm{H} / \mathrm{R}$ contributed to activation of TGF- $\beta$ signaling and the occurrence of oxidative stress in PC-12 cells; and ALK5 inhibition or knockdown significantly alleviated the effect of H/R on PC-12 cells. We found that both inhibition and knockdown of ALK5 decreased SMAD2/3 phosphorylation and NOX2/4 expression, but ALK5 inhibitor had no effect on the expression of ALK5. The present study indicated that TGF- $\beta$ signaling activation exacerbated brain injury and was associated with the regulation of NOX2/4 expression.

Oxidative stress is characterized by the overproduction of ROS, which can induce mitochondrial DNA mutations, damage the mitochondrial respiratory chain, alter membrane permeability, and influence $\mathrm{Ca}^{2+}$ homeostasis and mitochondrial defense systems. This is a common event in the pathology of neurodegenerative disorders such as Alzheimer's disease, Parkinson's disease, Huntington's disease, amyotrophic lateral sclerosis, and multiple sclerosis, as well as ischemic stroke [6,17-19]. Generally, ROS are generated endogenously from molecular oxygen by cellular oxidases, mono- and di-oxygenases of the mitochondrial electron chain transport system, or peroxidases, and are involved in nerve cell damage after I/R injury. The major ROS involved in oxidative stress include superoxide anion, hydrogen peroxide, and hydroxyl radicals [20]. Multiple sources and processes lead to ROS generation in cells, such as NOX, nitric oxide synthase, cytochrome P-450, cyclooxygenase, lipoxygenase, and xanthine oxidase $[21,22]$; however, NOX is considered the primary enzyme responsible for ROS generation. To date, seven subtypes of NOX have been found in rats, namely, NOX1NOX5, dual oxidase 1 (DUOX1), and DUOX2, but only NOX2 and NOX4 were demonstrated as the major types involved in brain tissue ROS generation. In this study, we found that I/R injury markedly increased the expression of NOX2/4 and the total NOX enzyme activity in brain tissue [5]. Consistently, oxidative burst and increased tissue damage were found after cerebral I/R. In addition, there is considerable evidence, such as NOX inhibitor and knockout studies, demonstrating that NOX2 and NOX4 are the principal NOX isoforms involved in mediating oxidative stress and brain injury following cerebral ischemia [23]. Taken together, the above findings indicate that NOX2 and NOX4 play an important role in cerebral I/R injury.

To date, many studies have implicated various mechanisms in NOX expression regulation, such as transcription factors (NF- $\mathrm{KB}, \mathrm{AP}-1$, STAT1/3, and C/EBP), nuclear receptors (PPAR $\alpha$ ), and epigenetic regulation (histone acetylation and miRNAs) [24]. Nevertheless, the detailed mechanism for NOX expression regulation has not been fully elucidated. Tumor suppressor TGF- $\beta$ was recently considered an important factor in ROS generation $[15,25]$, but its involvement in NOX2/NOX4 expression regulation has not been fully clarified. Recent studies have reported that TGF- $\beta$ can induce ROS generation and the underlying mechanism is its role in the TGF- $\beta$ /ALK5/SMAD2/3, MAPK, and JNK signaling pathways [12]. The canonical 
TGF- $\beta$ signaling pathway molecules comprise TGF- $\beta$ s, TGF- $\beta$ receptors (ALK1, ALK5, etc.), and SMAD1-SMAD8 [13]. Regarding ALK5 for example, TGF- $\beta$ signaling activation involves in the following processes: TGF- $\beta$ binding to ALK5 forming a complex with kinase activity, then the activated kinase phosphorylates the downstream substrate SMAD2/3, then with the help of SMAD4, phosphorylated SMAD2/3 translocates into nuclear and promote gene expression [13]. Emerging evidence has demonstrated that TGF- $\beta$ signaling is crucial in the pathogenesis of several CNS disorders, such as neurodegenerative disorders and ischemic stroke $[14,15]$. Previous studies have found that increased TGF- $\beta$ levels was correlated with cerebral ischemic injury [16]. In this study, we also found that TGF- $\beta$ signaling was positively correlated with the expression of NOX2/NOX4, as well as ROS generation following cerebral ischemia/reperfusion injury. Thus, we speculated that TGF- $\beta$ signaling is likely responsible for NOX2/NOX4 expression regulation and ROS generation. This was confirmed in our present study where inhibition of ALK5 decreased the phosphorylation of SMAD2/3, expression of NOX2/NOX4, and the production of ROS in vivo or in vitro. However, Sb505124 (a specific inhibitor of ALK5) had no effect on the expression of ALK5. Unlike the inhibition of ALK5, siRNA knockdown of ALK5 not only significantly decreased the expression of ALK5, but also decreased the phosphorylation of SMAD2/3 and the expression of NOX2/NOX4. This suggested that the TGF- $\beta$ signaling pathway can be activated independent of the increased expression of ALK5 i.e. in an ALK5 expression-dependent manner. These results suggested that the phosphorylation of SMAD2/3 will result in overexpression of NOX2/NOX4 and oxidative burst.

To our knowledge, excessive ROS causes damage to biological macromolecules such as ALDH2, a mitochondrial enzyme responsible for the oxidation of reactive aldehydes (high toxicity) formed during the process of I/R injury to carboxylic acids (low toxicity) [26], causing extensive damage to tissues or organs. Thus, it may be inferred that ROS scavenging is a key method for oxidative stress treatment. In fact, previous clinical and experimental data showed that reduced ROS generation inhibited brain damage; for instance, the NOX2 selective inhibitor gp91ds-tat significantly attenuated NOX enzyme activity and was strongly neuroprotective [27]. Many cerebral ischemia studies have found that apocynin, a nonisoform-specific NOX inhibitor, has been shown to reduce superoxide increase, oxidative stress, post-ischemic inflammation, and infarct volume, and improved neurological function in focal cerebral ischemia animal models [28-30]. Also, NOX2 or NOX4 knockout animals have been demonstrated to have reduced ROS and oxidative stress in the brain after focal cerebral ischemia [29]. These suggested NOX2 and NOX4 as potential targets for antioxidant therapy. In this present study, we found that high expression of NOX2/NOX4 was associated with increased ROS generation and low expression of NOX2/NOX4 was associated with decreased ROS generation, consistent with other research findings. Thus, any influence on the expression of NOX2/NOX4 or the enzyme activity of NOX can be used for ischemic stroke therapy. Because the TGF- $\beta$ signaling pathway plays an important role in NOX2 and NOX4 expression, it is feasible that oxidative stress can be alleviated via inhibition of TGF- $\beta$ signaling.

Summarily, this study is the first to explore the relationship between TGF- $\beta$ signaling and ROS generation, and demonstrated that the underlying mechanism involved is the TGF- $\beta /$ ALK5/SMAD(2/3)/NOX(2/4) axis, which will enrich the theoretical basis underlying the pathogenesis of cerebral I/R oxidative stress injury and provide experimental evidence. Based on our hypothesis, TGF- $\beta$ signaling may be a novel target for ischemic stroke antioxidant treatment.

\section{Abbreviations}

I/R (cerebral ischemia/reperfusion injury); NOX (NADPH oxidase); ROS (reactive oxygen species); H/R (hypoxia/reoxygenation); ALK5 (activin receptor-like kinase); TGF- $\beta$ (transformation growth factor beta). 


\section{Acknowledgements}

This work was supported by the National Natural Science Foundation of China (No. 81603107 to Zhong-Bao Yang and No.81600040 to Ai-Ping Wang), Health and Family Planning Commission of Hunan Province (No. B2016160 to Mei-Ling Zuo), the Education Department of Hunan Province (No.15C0161 to Zheng-Lou), the Zhengxiang Scholar Program (Prof. XiangYang Tang) of the University of South China, The Construction Program of the Key Discipline in Hunan Province, and the Affiliated Changsha Hospital of Hunan Normal University.

\section{Disclosure Statement}

No conflict of interests exists.

\section{References}

1 Tsai CF, Thomas B, Sudlow CL: Epidemiology of stroke and its subtypes in Chinese vs white populations: a systematic review. Neurology 2013;81:264-272.

2 Wu P, Zuo X, Ji A. Stroke-induced microRNAs: The potential therapeutic role for stroke. Exp Ther Med 2012;3:571-576.

-3 Ying W, Xiong ZG: Oxidative stress and NAD in ischemic brain injury: current advances and future perspectives. Curr Med Chem 2010;17:2152-2158.

-4 Jiang YF, Liu ZQ, Cui W, Zhang WT, Gong JP, Wang XM, Zhang Y, Yang MJ: Antioxidant effect of salvianolic acid B on hippocampal CA1 neurons in mice with cerebral ischemia and reperfusion injury. Chin J Integr Med 2015;21:516-522.

-5 Chen H, Yoshioka H, Kim GS, Jung JE, Okami N, Sakata H, Maier CM, Narasimhan P, Goeders CE, Chan PH: Oxidative stress in ischemic brain damage: mechanisms of cell death and potential molecular targets for neuroprotection. Antioxid Redox Signal 2011;14:1505-1517.

6 Ma MW, Wang J, Zhang Q Wang R, Dhandapani KM, Vadlamudi RK, Brann DW: NADPH oxidase in brain injury and neurodegenerative disorders. Mol Neurodegener 2017;12:7.

7 Bedard K, Krause KH: The NOX family of ROS-generating NADPH oxidases: physiology and pathophysiology. Physiol Rev 2007;87:245-313.

-8 Zhang HF, Li TB, $\quad$ Liu B, Lou Z, Zhang JJ, Peng JJ, Zhang XJ, Ma QL, Peng J, Luo XJ: Inhibition of myosin light chain kinase reduces NADPH oxidase-mediated oxidative injury in rat brain following cerebral ischemia/reperfusion. Naunyn Schmiedebergs Arch Pharmacol 2015;388:953-963.

-9 Zhang YS, Liu B, Luo XJ, Li TB, Zhang JJ, Peng JJ, Zhang XJ, Ma QL, Hu CP, Li YJ, Peng J, Li Q: Nuclear cardiac myosin light chain 2 modulates NADPH oxidase 2 expression in myocardium: a novel function beyond muscle contraction. Basic Res Cardiol 2015;110:138.

-10 Weiss A, Attisano L: The TGF-beta superfamily signaling pathway. Wiley Interdiscip Rev Dev Biol 2013;2:47-63.

11 Massagué J: How cells read TGF- $\beta$ signals. Nat Rev Mol Cell Biol 2000;1:169-178.

12 Hagler MA, Hadley TM, Zhang H, Mehra K, Roos CM, Schaff HV, Suri RM, Miller JD: TGF- $\beta$ signalling and reactive oxygen species drive fibrosis and matrix remodelling in myxomatous mitral valves. Cardiovasc Res 2013;99:175-184

13 Wang W, Rigueur D, Lyons KM: TGF- $\beta$ signaling in cartilage development and maintenance. Birth Defects Res C Embryo Today 2014;102:37-51

14 Gomes FC, Sousa, Vde O, Romão L: Emerging roles for TGF- $\beta 1$ in nervous system development. Int J Dev Neurosci 2005;23:413-424.

15 Hsieh HL, Wang HH, Wu WB, Chu PJ,Yang CM: Transforming growth factor- $\beta 1$ induces matrix metalloproteinase- 9 and cell migration in astrocytes: roles of ROS-dependent ERK-and JNK-NF- $\kappa B$ pathways. J Neuroinflammation 2010;7:88. 
Lou et al.: A New Mechanism for NOX2 and NOX4 Expression Regulation in the Process of Cerebral Ischemia/Reperfusion Injury

16 Vivien D, Ali C: Transforming growth factor- $\beta$ signalling in brain disorders. Cytokine Growth Factor Rev 2006;17:121-128.

17 Bennett DA, Krishnamurthi RV, Barker-Collo S, Forouzanfar MH, Naghavi M, Connor M, Lawes CM, Moran AE, Anderson LM, Roth GA, Mensah GA, Ezzati M, Murray CJ, Feigin VL: The global burden of ischemic stroke: findings of the GBD 2010 study. Glob Heart 2014;9:107-112.

18 Kowal SL, Dall TM, Chakrabarti R, Storm MV, Jain A: The current and projected economic burden of Parkinson's disease in the United States. Mov Disord 2013;28:311-318.

19 Guo C, Sun L, Chen X, Zhang D: Oxidative stress, mitochondrial damage and neurodegenerative diseases. Neural Regen Res 2013;8:2003-2014.

20 Grivennikova, VG, Vinogradov AD: Partitioning of superoxide and hydrogen peroxide production by mitochondrial respiratory complex I. Biochim Biophys Acta 2013;1827:446-454.

21 Maraldi T: Natural compounds as modulators of NADPH oxidases. Oxid Med Cell Longev 2013;2013:271602.

22 Shyu KG, Chang CC, Yeh YC, Sheu JR, Chou DS: Mechanisms of ascorbyl radical formation in human plateletrich plasma. Biomed Res Int 2014;2014:614506.

-23 Kleinschnitz C, Grund H, Wingler K, Armitage ME, Jones E, Mittal M,Barit D, Schwarz T, Geis C, Kraft P, Barthel K, Schuhmann MK, Herrmann AM, Meuth SG, Stoll G, Meurer S, Schrewe A, Becker L, Gailus-Durner V, Fuchs H, Klopstock T, de Angelis MH, Jandeleit-Dahm K, Shah AM, Weissmann N, Schmidt HH: Post-stroke inhibition of induced NADPH oxidase type 4 prevents oxidative stress and neurodegeneration. PLoS Biol 2010;8.pii: e1000479.

24 Manea SA, Constantin A, Manda G, Sasson S, Manea A: Regulation of Nox enzymes expression in vascular pathophysiology: focusing on transcription factors and epigenetic mechanisms. Redox Biol 2015;5:358366.

-25 Ghatak S, Hascall VC, Markwald RR, Feghali-Bostwick C, Artlett CM, Gooz M, Bogatkevich GS, Atanelishvili I, Silver RM: TGF beta-1 induced CD44v6-NOX4 signaling in pathogenesis of idiopathic pulmonary fibrosis. J Biol Chem 2017;292:10490-10519.

26 Glatt H, Rost K, Frank H, Seidel A, Kollock R: Detoxification of promutagenic aldehydes derived from methylpyrenes by human aldehyde dehydrogenases ALDH2 and ALDH3A1. Arch Biochem Biophys 2008;477:196-205.

27 Rey FE, Cifuentes ME, Kiarash A, Quinn MT, Pagano PJ: Novel competitive inhibitor of NAD (P) H oxidase assembly attenuates vascular 02- and systolic blood pressure in mice. Circulation research 2001;89:408414.

28 Chen H, Song YS, Chan PH: Inhibition of NADPH oxidase is neuroprotective after ischemia-reperfusion. J Cereb Blood Flow Metab 2009;29:1262-1272.

29 Jackman KA, Miller AA, De Silva TM, Crack PJ, Drummond GR, Sobey CG: Reduction of cerebral infarct volume by apocynin requires pretreatment and is absent in Nox2-deficient mice. Br J Pharmacol 2009;156:680-688.

-30 Tang XN, Cairns B, Cairns N, Yenari MA: Apocynin improves outcome in experimental stroke with a narrow dose range. Neuroscience 2008;154:556-562. 